\title{
Status of the Jefferson Lab Polarized Beam Physics Program and Preparations for Upcoming Parity Experiments
}

\author{
J. Grames, P. Adderley, M. Baylac, J. Clark, A. Day, \\ J. Hansknecht, M. Poelker, M. Stutzman \\ Thomas Jefferson National Accelerator Facility, \\ 12000 Jefferson Avenue, Newport News, VA 23606, USA
}

\begin{abstract}
An ambitious nuclear physics research program continues at Jefferson Lab with Users at three experiment halls receiving reliable, highly polarized electrons at currents to $100 \mu \mathrm{A}$. The polarized photoguns and drive lasers that contribute to Jefferson Lab's success will be described as well as significant events since PES2000. Typical of conditions at accelerators worldwide, success brings new challenges. Beam quality specifications continue to become more demanding as Users conduct more challenging experiments. In the months that follow this workshop, two parity violation experiments will begin at Jefferson Lab, G0 and HAPPEx2. The photogun requirements for these experiments will be discussed as well as our plans to eliminate/minimize systematic errors. Recent efforts to construct high power Ti-Sapphire drive lasers for these experiments also will be discussed.
\end{abstract}

\section{POLARIZED PHOTOINJECTOR}

The Jefferson Lab photoinjector delivers highly polarized electrons to three Users simultaneously at beam currents spanning six decades $(100 \mathrm{pA}$ to $100 \mu \mathrm{A})$. Two identical $-100 \mathrm{kV}$ DC photoemission electron guns (see Figure 1) provide all of the electrons for the nuclear physics program at the laboratory. One is used for production beam delivery to Users and the second serves as a spare. We use strained layer GaAs photocathodes from Bandwidth Semiconductor (formerly Spire Corporation). These GaAs-on-GaAsP photocathodes are grown to the SLAC specification and the best samples provide beam polarization 70 to $80 \%$. Cathode preparation entails brief ( 15 minute) atomic hydrogen cleaning in a dedicated chamber after which the cathode is transferred under nitrogen purge and bath to the gun chamber which is then baked. Subsequently, the cathode is heated and then activated with cesium and nitrogen trifluoride to establish negative electron affinity (NEA). The guns and past performance are further described in ref. [1,2].

Two laser tables straddle the beamline and provide a direct optical path to either gun. The drive lasers emit rf-pulsed light with picosecond pulsewidths, synchronous to the CEBAF accelerating frequency $(1.497 \mathrm{GHz})$. A variety of lasers and rf pulse repetition rates can be chosen to meet the demands of the physics program which varies on a monthly basis. Diode laser systems [3] have been used to satisfy most of 
the beam current needs of the physics Users. Diode lasers are reliable and relatively maintenance free and can be easily configured to produce different repetition rates; $499 \mathrm{MHz}$ for beam to a single experiment hall and $1497 \mathrm{MHz}$ for beam to all three halls. The demand for both high current and high polarization has spurred our development of high power Ti-Sapphire drive lasers with high repetition rates [4]. A new parity violation experiment at Hall $\mathrm{C}(\mathrm{G} 0)$ has extended the need for drive laser flexibility by requiring beam with a pulse repetition rate of $31.1875 \mathrm{MHz}$. To accommodate this experiment we have developed a variety of home made modelocked lasers and we also have purchased a commercial laser. The optical pulse width of all of the CEBAF drive lasers is typically 70 ps which is compatible with the preaccelerator phase acceptance of the photoinjector. The laser beams are focused at the photocathode to a spot diameter of 500 to 700 microns.

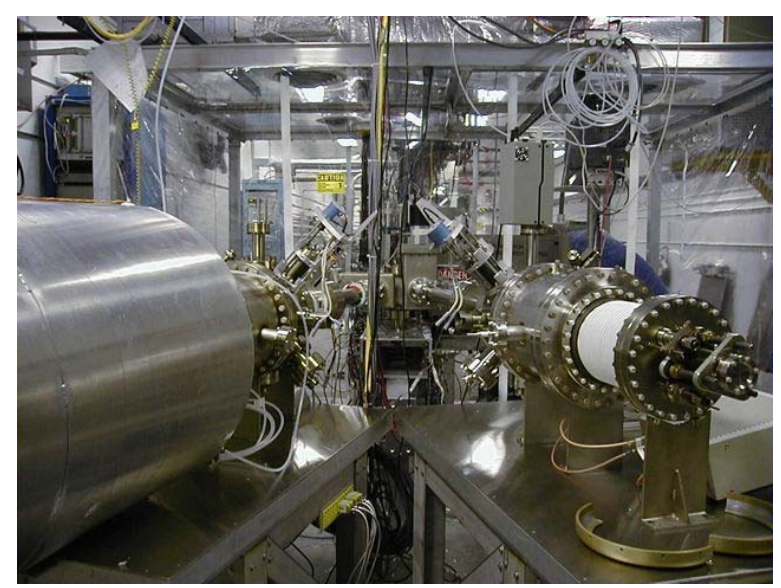

FIGURE 1. The two CEBAF photoemission guns (the gun at left is covered with a "shroud" to prevent accidental contact with high voltage). The guns are identical and positioned symmetrically on either side of a 15 degree bend magnet. The polarity of the bend magnet is flipped to switch between guns. A temperature controlled, plastic-walled laser hut is shown in the background.

\section{SOURCE PERFORMANCE}

Over the course of years, we have refined our photocathode preparation procedures to obtain consistent results; namely, high quantum efficiency and high polarization [M. Baylac et al., these proceedings]. Typical values of quantum efficiency and polarization at two standard operating wavelengths are $\sim 0.15 \% \mathrm{QE}$ at $840 \mathrm{~nm}$ for polarization $>70 \%$ and $\sim 1.0 \%$ QE at $770 \mathrm{~nm}$ for polarization $\sim 30 \%$.

The operating lifetime of the CEBAF polarized photoguns is very good. At high current $(250 \mu \mathrm{A}$ extracted gun current), the $1 / \mathrm{e}$ photocathode lifetime is approximately $300 \mathrm{C}$. At lower current $(<100 \mu \mathrm{A})$, lifetime increases to approximately $600 \mathrm{C}$. The endstations receive roughly $90 \%$ of the extracted gun current; the rest is dumped on apertures that limit the transverse and longitudinal acceptance of the injector.

We attribute our excellent operating lifetime to low vacuum pressure within the guns. Pressure in the $10^{-12}$ Torr range is obtained using an array of non-evaporable getter pumps (NEG) surrounding the cathode/anode gap. The beamline downstream 
of the gun activation chamber is also coated with NEG material. Vacuum quality is maintained during beam delivery by eliminating unintentional photoemission from portions of the photocathode that are not directly illuminated with light from the drive lasers. Electrons that originate from the edge of the photocathode can have extreme trajectories, eventually hitting the vacuum chamber walls, liberating gas and degrading vacuum pressure. The $\mathrm{QE}$ of the edge of the photocathode is killed using the process of anodization. These features and techniques are described in past Polarized Source Workshop proceedings.

Gun maintenance and associated "downtime" varies according to the nuclear physics program and User demands for current. At high current ( $250 \mu \mathrm{A}$ extracted gun current), we can deliver uninterrupted beam for 2 to 3 weeks before we are forced to move the laser to a fresh photocathode location. We can move the laser spot approximately 5 times before the photocathode must be heated and reactivated to restore $\mathrm{QE}$. The process of heating and reactivating takes 8 hours and $\mathrm{QE}$ restores to initial values. At lower gun currents, QE degrades more slowly and beam interruptions occur less frequently. An example of a particularly long period of uninterrupted beam delivery occurred during the summer months of 2001, when we delivered beam (60 $\mu \mathrm{A}$ gun current) to three experiments for three months from one photocathode location.

Unfortunately, limited experience suggests catastrophic field emission develops within each gun after approximately four activations. Field emission necessitates cathode electrode re-polishing and the installation of a new photocathode sample, a time consuming, labor intensive process. A load lock gun is being developed which eliminates the need to apply cesium on the photocathode within the high voltage chamber [M. Stutzman, these proceedings]. By keeping cesium from the cathode electrode, we hope to eliminate field emission from our guns.

\section{DRIVE LASER}

The drive laser system is the most dynamic component of our polarized photoinjector. Three Users make for a dynamic physics program that requires the laser table be easily configured to achieve different combinations of beam intensity (laser power), electron polarization (laser wavelength), RF synchronization (1.497 GHz or sub-harmonic) and high availability (little maintenance). Other important laser features include cost and availability (sometime diode laser vendors take a product off the market), intensity noise at the helicity reversal rate, and the extent that each laser turns off between optical pulses (so that one laser does not inadvertently provide beam to all three halls).

RF-synchronous gain-switched diode lasers are easy to use, low maintenance, and reliable. The intensity noise at the helicity reversal rate is very good, $<0.1 \%$. However, the diode laser power is low $(<100 \mathrm{~mW})$ and the laser wavelength is fixed for either high current $(770 \mathrm{~nm})$ or high polarization $(840 \mathrm{~nm})$. Additionally, amplified spontaneous emission produces DC beam that leaks into the RF buckets for other Users. For high current Users this is not problematic, but DC leakage to low current (nanoamp) Users can cause polarization dilution. Finally, the vendor Spectra Diode 
Labs no longer sells the diode optical amplifier used to boost laser power from the gain switched seed laser. We are testing another vendor (Toptica) although the lifetime performance appears questionable.

An alternative to gain switched diode lasers is the Ti-Sapphire laser that can provide both high current and high polarization. The laser power exceeds $300 \mathrm{~mW}$ and the wavelength is tunable from 770 to $860 \mathrm{~nm}$, which means the laser can be used for different photocathodes with peak polarization occurring at different wavelengths. These desirable features (i.e., high power and tunable wavelength) come with the cost of added complexity and more frequent need for maintenance (i.e., mirrors must be cleaned and realigned). The Ti-Sapphire laser can also provide the low pulse repetition rate for the $\mathrm{G} 0$ experiment $(31.1875 \mathrm{MHz})$, something not achievable with diode lasers and the method of gain switching.

\section{HELICITY CORRELATED SYSTEMATIC CONTROL}

Any parameter of the beam (e.g., intensity, position, or energy) that may change when the laser helicity is reversed is called a helicity correlated (HC) effect. To satisfy beam requirements for the parity violation experiments G0 and HAPPEx2, the accelerator must provide beam with integrated $\mathrm{HC}$ intensity variation below one part per million and $\mathrm{HC}$ position variation at the target less than one nanometer.

To first order, the sources of helicity correlated effects originate at the Pockels cell and the strained layer photocathode. Ref. [5] describes in great detail the various factors that contribute to different helicity correlated effects. In this paper, only the feedback controls employed at JLab are described.

JLab multi-User operation imposes constraints on the laser configuration and choice of optical elements used to control HC systematics. The most stringent are those imposed on the optical elements common to all lasers. These elements include transport mirrors, a Pockels cell for creating/reversing the circular polarization, an insertable half-wave plate for systematic reversal, and a rotatable half-wave plate used to null the effect of QE anisotropy of the high polarization photocthodes. During the parity violation HAPPEx experiment the Pockels cell was used to control intensity asymmetry using the PITA effect. The rotating half-wave plate was used to minimize HC position sensitivity. Active HC position feedback was not performed. However, these common optical elements affect all Users.

The demands for the upcoming Hall C (G0) and Hall A (HAPPEx2) experiments require the capability to independently control $\mathrm{HC}$ intensity and position for each parity User. This meant adding additional parity controls specific to each laser system. Independent intensity control was achieved with an Intensity Attenuator (IA), a low voltage Pockels cell inserted between two similarly oriented optical polarizers after the laser, but prior to the combining optic. The cell has a low insertion loss $(\sim 10 \%)$ and is very compact, necessary for installing into the region prior to the laser combination optic. The cell voltage is remotely controlled by a DAC and is modulated with the helicity reversal sequence. The transmitted intensity is sinusoidal with applied voltage and has maximum transmission at zero voltage. To shift the sinusoid achieving linear, bipolar intensity modulation, and to operate at a non-biased cell voltage, a tenth-order 
waveplate is inserted upstream of the cell. The resultant sensitivity of the IA has been measured to be $\sim 300 \mathrm{ppm} /$ volt.

To achieve independent position control we retrofit some of our remotely controlled picomotor mirror mounts upstream of the combination optic with kinematic mounts containing piezoelectric (PZT) stacks. A PZT stack voltage is set remotely by a DAC and is modulated with the helicity reversal sequence providing deflection of the laser beam toward the cathode. The result is an electron beam modulated in position from the photocathode. An IA and PZT have been added to the optical paths for both parity lasers. The G0 experiment demonstrated benchmark capability in September 2002 with these controls in place.

Finally, a software lock has been implemented to manage the interface between measurement of helicity correlated beam parameters at the experimental targets and actuation of the parity controls on the laser table. It is worth noting that the injector and accelerator diagnostics detect all three beams and therefore we use only diagnostics located near the experimental targets for actuating the parity controls.

\section{TI-SAPPHIRE LASER DEVELOPMENT}

The time-of-flight proton resolution of the G0 detector requires that the experiment operate at the $48^{\text {th }}$ sub-harmonic $(31.1875 \mathrm{MHz})$ of the accelerator fundamental. As noted, this repetition rate is not suited for diode lasers. Instead, a home built TiSapphire laser was built at JLab using a 4-mirror folded cavity design and a solid state green pump laser (Coherent Verdi-10). Despite an overall cavity length of $4.84 \mathrm{~m}$, the laser fits on a $1.2 \mathrm{~m} \mathrm{x} 0.25 \mathrm{~m}$ optical breadboard. An acousto-optic modulator at $15.59375 \mathrm{MHz}$ is used to actively mode-lock the laser. The laser power is $>300 \mathrm{~mW}$ at $825 \mathrm{~nm}$ with $<0.2 \%$ intensity noise at the helicity reversal rate. However, the optical pulse length of 180 ps FWHM presented difficulties for pre-accelerator transmission.

In parallel, a commercial Ti-Sapphire laser was purchased from Time-Bandwidth. This laser uses a passive SESAM mode-locking technique. The performance of the laser has been turn-key with little maintenance. The output power is $>300 \mathrm{~mW}$ at 840 $\mathrm{nm}$ and is tunable from 770 to $860 \mathrm{~nm}$. The measured pulse length is $70 \mathrm{ps} \mathrm{FWHM}$ and provides adequate injector transmission. Importantly, the phase noise is $\sim 1 \mathrm{ps}$ and the $\mathrm{HC}$ intensity noise at $30 \mathrm{~Hz}$ has been measured between 0.1 and $0.02 \%$. This laser is presently installed in the tunnel and is being used for the G0 experiment.

Finally, the HAPPEx2 experiment, to begin in early 2003, requires a high polarization, high current $(>80 \mu \mathrm{A})$ electron beam at $499 \mathrm{MHz}$. A second laser operating at this repetition rate has been ordered from Time-Bandwidth.

\section{ACKNOWLEDGMENTS}

We thank members of the G0 and Hall A Parity collaborations for continued effort developing the laboratory's parity controls. Brian Bevins contributed substantially by 
writing the parity control software locks. This work was supported by the USDOE under contract DE-AC05-84ER40150.

\section{REFERENCES}

1. Poelker, M., et al., "Polarized Source Performance and Developments at Jefferson Lab" in $14^{\text {th }}$ International Spin Physics Symposium, edited by K. Hatanaka et al., AIP Conference Proceedings 570, New York: American Institute of Physics, 2001, pp. 943-948.

2. See contribution by M. Stutzman, same proceedings.

3. Poelker, M., Appl. Phys. Lett. 67, 2762 (1995).

4. Poelker, M., and Hansknecht, J., "A High Power and High Repetition Rate Modelocked TiSapphire Laser for Photoinjectors" in Proceedings of the 2001 Particle Accelerator Conference, edited by P. Lucas et al., IEEE Catalog Number 01CH37268C, 2001, pp. 95-97.

5. Humensky, T.B., et al., "SLAC's Polarized Electron Source Laser and Optics Systems and Minimization of Helicity Correlations for the E-158 Parity Violation Experiment", Submitted to Nucl. Instrum. Meth. A (2002) and SLAC-PUB-9381 (2002). 